\title{
Outlier Analysis of Categorical Data Using Infrequency
}

\author{
M. Krishna Murthy, A. Govardhan, Lakshmi SreenivasaReddy D \\ Research scholar, ANU, Guntur, India \\ Krishna_mudimbi@yahoo.co.uk \\ Director of Evaluation, JNTUH, Hyderabad, India \\ govardhan_cse@jntuh.ac.in \\ Department of CSE, Rise Gandhi Groups of Institutions, Ongole, India. \\ urdlsreddy@yahoo.com.
}

\begin{abstract}
Anomalies are those objects, which will act with different behavior and do not follow with the remaining records in the databases. Detecting anomalies is an important issue in many fields. Though many methods are available to detect anomalies in numerical datasets, only a few methods are available for categorical datasets. In this work, a new method has been proposed. This algorithm finds anomalies based on infrequent itemsets in each record. These outliers are generated by Apriori property on each record values in datasets. Previous methods may not distinguish different records with the same frequency. These give same score for each record. For each record a score is generated based on infrequent itemsets which is called MAD score in this paper. This algorithm utilizes the frequency of each value in the dataset. FPOF method is used the concept of frequent itemset and otey method used infrequent itemset. But these cannot distinguish records perfectly. The proposed algorithm has been applied on Nursery dataset and Bank dataset taken from "UCl Machine Learning Repository". Numerical attributes are excluded from Datasets for this analysis. The experimental results show that it is efficient for outlier detection in categorical dataset.
\end{abstract}

\section{Indexing terms/Keywords}

Data Mining, Outlier detection, FPOF score, FDOD Score, MAD score

\section{Academic Discipline and Sub-Disciplines}

Computer Science and Engineering

\section{SUBJECT CLASSIFICATION}

\section{Data Mining}

\section{TYPE (METHOD/APPROACH)}

Outlier Analysis.

\section{Council for Innovative Research}

\author{
Peer Review Research Publishing System
}

\section{Journal: INTERNATIONAL JOURNAL OF COMPUTERS \& TECHNOLOGY}

Vol 8, No 3 


\section{INTRODUCTION}

Outlier analysis is an important research field in many fields like networks, medicine and Business decisions. This analysis concentrates on detecting infrequent data records in dataset. Most of the existing systems concentrate on numerical attributes or ordinal attributes and sometimes, categorical attribute values can be converted into ordinal values there to categorical values. This process is not always preferable. This paper presents a novel method for finding anomalies in categorical data. The mechanism in the previous methods which are depends on frequent itemsets is that, these calculates frequency of each value in each records and checked with the threshold value, whether that value frequent or not. Then they formed all combinations of itemsets and find their frequency by scanning the dataset. All these combinations are the subsets of records. Based on infrequent itemsets their respective scores are generated. Top $k$ outliers are selected based on the least k-scores. The parameter s used in this method ' $k$ ', the number of outliers and a threshold value ' $\sigma$ ' to decide frequent item sets in each data object' [1].

\section{TERMINOLOGY}

Table.1. Terminology used in this paper.

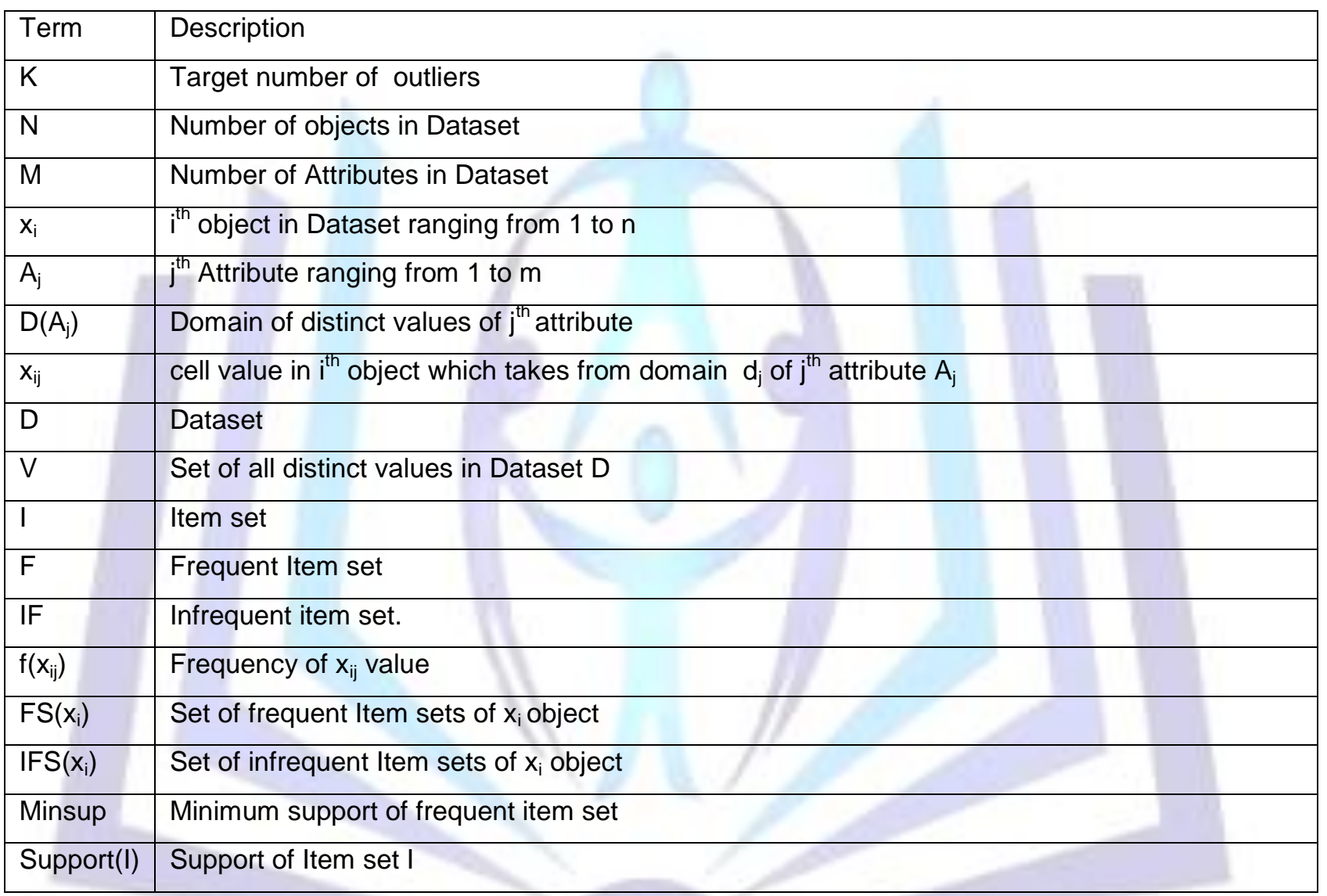

\section{Some of the Existing Approaches for Categorical Datasets based on Item Frequency Frequent Pattern Outlier Factor (FPOF) algorithm:}

This algorithm utilizes the Apriori algorithm as a first step to find all frequent Item sets. This method needs a human defined threshold value called" minimum support" ' $\sigma$ ' as input to find frequent item sets. By taking this threshold value, it makes all combinations of values of each record and compares the frequency of each combination with threshold value and finds each combination whether it is frequent or not. To find frequency of each combination, it needs one scan of the dataset. The formula utilized in this algorithm is

$$
\text { FPOFScore }=\sum_{F \subset x i \wedge F \in I F\left(x_{i}\right)} \frac{\operatorname{support}(F)}{\left|F S\left(x_{i}\right)\right|}
$$

Where Dataset $D=\{A 1, A 2-------A m\}$,

Minimum support $=$ ' $\sigma$ ',

Number of outliers $=$ ' $k$ ', 
Where $\mathrm{F}$ is the frequent item set which satisfies the minimum support,

$F S\left(x_{i}\right)$ is the set of all frequent itemsets which are subsets of the record " $x_{i}$ ",

This model finds FPOF score for each record and selects k-outliers as least k-scores. If there is no frequent itemset at all in any record, identifying the score is a problem in this method.

\section{a) Fast Distributed Outlier Detection(FDOD) (Otey) Algorithm}

This algorithm also used the concept of frequent pattern method. The inverse Apriori is used in this model which say that "every super set of an infrequent itemset is again an infrequent set. So that, model reduces number of scannings of the dataset. This model first finds all combinations of subsets of each record and checks its support with threshold ' $\sigma$ '.

It considers infrequent item sets and finds the FDOD score for each record by the below formula,

$$
\text { FDODScore }=\sum_{I F \subset x_{i} \wedge I F \in I F S\left(x_{i}\right)} \frac{1}{\left|I F S\left(x_{i}\right)\right|}
$$

Where IF is the infrequent item set which does not satisfy the minimum support,

IFS $\left(x_{i}\right)$ is the set of all infrequent itemsets which are subsets of the record " $x_{i}$ ",

This model finds FDOD score for each record and selects k-outliers as top k-scores. If there is no infrequent itemset at all in any record, then identifying the score is another problem in this method

\section{b) Attribute Value Frequency (AVF) algorithm}

This algorithm is simple for finding scores of each object. and faster approach to detect outliers that minimizes the scans over the data and does not need to create more space and more search for combinations of attribute values or item sets is Attribute Value Frequency (AVF) algorithm. An outlier point " $x_{i}$ " is defined based on the AVF Score below:

$$
\text { AVFScore }=\frac{1}{m} \sum_{i=1}^{m} \frac{f\left(x_{i j}\right)}{n}
$$

Where $f\left(x_{i j}\right)$ is the frequency of each value involved in each record, ' $m$ ' is the number of attributes,

' $n$ ' is the dataset size,

" $\mathrm{X}_{\mathrm{ij}}$ " is the cell value in $\mathrm{i}^{\text {th }}$ record and $\mathrm{j}^{\text {th }}$ Attribute.

This model finds AVF score for each record and selects k-outliers as least k-scores. When these above algorithms applied on a sample data below with threshold value $=5$, one problem is identified.

Table.2. Comparison of existing model scores on Sample Health data.

\begin{tabular}{|c|c|c|c|c|c|c|}
\hline S.No & Age & Income level & Disease & FPOF & FDOD & AVF \\
\hline 1 & Middle & Low & Cancer & 0.35 & 1.33 & 0.53 \\
\hline 2 & Middle & High & Cancer & 0.35 & 1.33 & 0.53 \\
\hline 3 & Young & Low & Cancer & 0.27 & 1.83 & 0.53 \\
\hline 4 & Middle & High & Cancer & 0.35 & 1.33 & 0.53 \\
\hline 5 & Young & Low & Sugar & 0.17 & 2.83 & 0.47 \\
\hline 6 & Young & Low & Sugar & 0.17 & 2.83 & 0.47 \\
\hline 7 & Middle & High & Cancer & 0.35 & 1.33 & 0.53 \\
\hline 8 & Young & Low & Sugar & 0.17 & 2.83 & 0.47 \\
\hline 9 & Middle & High & Cancer & 0.35 & 1.33 & 0.53 \\
\hline 10 & Young & High & Sugar & 0.17 & 2.83 & 0.47 \\
\hline
\end{tabular}


From all the above scores it is observed that some of the scores are same for different combination of values. If it is needed to select $10 \%$ of outliers which record will be selected? Since $5^{\text {th }}, 6^{\text {th }}$, 8 th and $10^{\text {th }}$ records scores are same, there exists some ambiguity. If it is compulsory, the $5^{\text {th }}$ may be selected because $5^{\text {th }}$ is the first one. By observing these four records $10^{\text {th }}$ one is very different. But the scores are same. The proposed model can distinguish this $10^{\text {th }}$ record which is different from the $5^{\text {th }}, 6^{\text {th }}$ and $8^{\text {th }}$ records.

\section{c) Proposed Model (MAD Score)}

This proposed algorithm also used the infrequent itemsets which are generated by Appriori concept. This proposed model finds the score for each record. We call this score as MAD score.

$$
\text { MADscore }=\frac{n}{1+\sum \text { Freq }(\text { Infrequentitemset })}=\frac{n}{1+\sum_{\forall I F \in I F S\left(x_{i}\right)} \sup (I F)}
$$

Where ' $n$ ' is the dataset length,

IF is the infrequent itemset,

IFS is the Set of Infrequent itemsets,

$x_{i}$ is the $i^{\text {th }}$ record in dataset.

Sup (IF) is Frequency of IF

From the below table it is revealed that $10^{\text {th }}$ record is the most different outlier, and then the records $5^{\text {th }} 6^{\text {th }} 8^{\text {th }}$ are in the next order. $5^{\text {th }} 6^{\text {th }} 8^{\text {th }}$ and $10^{\text {th }}$ records gave the same scores in FPOF, FDOD and AVF. But the proposed model distinguishes the $10^{\text {th }}$ record separately. Not only the only the $10^{\text {th }}$ record, for all different records are giving different scores by the proposed model. So the proposed model is reliable to find reliable outliers. The proposed model is applied on Bank Data set, Nursery Data set and Breast Cancer Dataset which are taken from $\mathrm{UCI} \mathrm{ML}$ repository [10].The comparison of results with FPOF, FDOD and AVF on Trail data is given below.

Table.3. Comparison of MAD score with FPOF, FDOD and AVF.

\begin{tabular}{|c|c|c|c|c|c|c|c|}
\hline S.No & Age & Income level & Disease & FPOF & FDOD & AVF & MAD \\
\hline 1 & Middle & Low & Cancer & 0.35 & 1.33 & 0.53 & 1.6666 \\
\hline 2 & Middle & High & Cancer & 0.35 & 1.33 & 0.53 & 1 \\
\hline 3 & Young & High & Cancer & 0.27 & 1.83 & 0.53 & 1.25 \\
\hline 4 & Middle & Low & Cancer & 0.35 & 1.33 & 0.53 & 1.6666 \\
\hline 5 & Young & Low & Sugar & 0.17 & 2.83 & 0.47 & 0.714 \\
\hline 6 & Young & Low & Sugar & 0.17 & 2.83 & 0.47 & 0.714 \\
\hline 7 & Middle & High & Cancer & 0.35 & 1.33 & 0.53 & 1 \\
\hline 8 & Young & Low & Sugar & 0.17 & 2.83 & 0.47 & 0.714 \\
\hline 9 & Middle & High & Cancer & 0.35 & 1.33 & 0.53 & 1 \\
\hline 10 & Young & High & Sugar & 0.17 & 2.83 & 0.47 & 0.8333 \\
\hline
\end{tabular}

\section{Experimental Results}

When the experiments are conducted on bank data with 45212 records by the proposed model, it has achieved the maximum classifier accuracy better than AVF. The experimental results are compared with AVF results because in previous research work AVF has given good results when compared with FPOF and FDOD. The bank data contains 7 variables and 46 values. The Bank Sample has partitioned into two parts, one is with "Yes" Class label (5299) and another is with "no" class label (39922 records) using Clementine tool. The "yes" label records are considered as outliers in this experiment. In this experiment $50 \%$ of outliers (2645 records) are selected randomly and mixed up with "no" class label. The mixed records (42567 records) considered for experiments. Both AVF and MAD models applied on the same built mixed records to delete top $100,200,300,400,500,600,700$ and 800 outliers. After deleted these outliers different classifiers are tested. The tested results are given bellow. 
Table.4. Comparison of classifier accuracies for Bank data (1-in-2 sample).

\begin{tabular}{|c|c|c|c|c|c|c|}
\hline Classifier & \multicolumn{2}{|c|}{ NN } & \multicolumn{2}{c|}{ LR } & \multicolumn{2}{c|}{ CHAID } \\
\hline $\begin{array}{c}\text { Number of } \\
\text { outliers } \\
\text { deleted }\end{array}$ & AVF Score & MAD Score & AVF Score & MAD Score & AVF Score & MAD Score \\
\hline 100 & $98.726 \%$ & $98.796 \%$ & $98.726 \%$ & $98.796 \%$ & $98.726 \%$ & $98.796 \%$ \\
\hline 200 & $98.763 \%$ & $98.865 \%$ & $98.763 \%$ & $98.865 \%$ & $98.763 \%$ & $98.865 \%$ \\
\hline 300 & $98.842 \%$ & $98.924 \%$ & $98.842 \%$ & $98.924 \%$ & $98.842 \%$ & $98.924 \%$ \\
\hline 400 & $98.904 \%$ & $98.991 \%$ & $98.904 \%$ & $98.991 \%$ & $98.904 \%$ & $98.991 \%$ \\
\hline 500 & $98.926 \%$ & $99.041 \%$ & $98.926 \%$ & $99.041 \%$ & $98.926 \%$ & $99.041 \%$ \\
\hline 600 & $98.966 \%$ & $99.117 \%$ & $98.966 \%$ & $99.117 \%$ & $98.966 \%$ & $99.117 \%$ \\
\hline 700 & $99.034 \%$ & $99.198 \%$ & $99.034 \%$ & $99.198 \%$ & $99.034 \%$ & $99.198 \%$ \\
\hline 800 & $99.082 \%$ & $99.208 \%$ & $99.082 \%$ & $99.208 \%$ & $99.082 \%$ & $99.208 \%$ \\
\hline
\end{tabular}

From the above results it is concluded that Classifiers built on the records when outliers are deleted by MAD Score algorithm gives better results when compared with AVF Score. Classifiers also applied on the original data without deleting outliers. These classifiers gave $88.302 \%$ only.

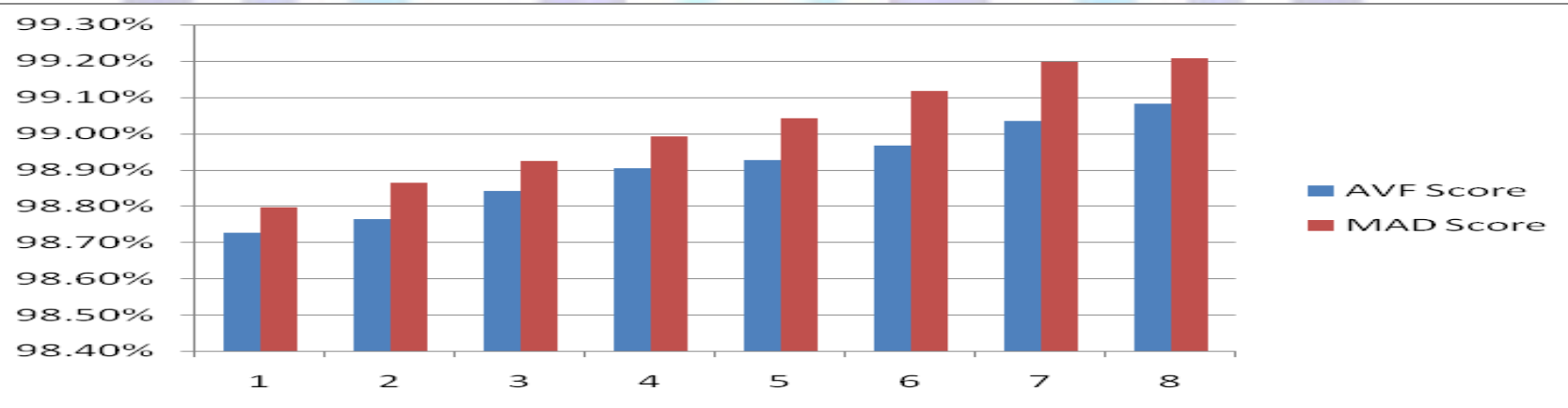

Fig.1.NN, LR and CHAID Classifiers accuracy when outliers deleted by AVF and MAD for below results

\section{Conclusion and Future work}

To sum up, this proposed method finds distinguished score for distinguishable records, where as the previous methods may not find different scores for different records. This model also gives reliable records as the classifiers get maximum accuracies when compared with old models. To form the combinations of item sets and scanning of the dataset for every itemset for frequency is a big problem in these models. Some possibility of solving this problem is parallel computing. When the attributes are increasing the complexity becomes more in these models.

\section{References}

[1] M. E. Otey, A. Ghoting, and and A. Parthasarathy, "Fast Distributed Outlier Detection in Mixed-Attribute Data Sets," Data Mining and Knowledge Discovery

[2] He, Z., Deng, S., Xu, X., "A Fast Greedy algorithm for Outlier mining" Proc. of PAKDD, 2006.

[3] I. S. Jacobs and C. P. Bean, "Fine particles, thin films and exchange anisotropy," in Magnetism, vol. III, G. T. Rado and H. Suhl, Eds. New York: Academic, 1963, pp. 271-350.

[4] P. Tan, M. Steinbach, and V. Kumar, Introduction to Data Mining: Pearson Addison-Wesley, 2005

[5] E. Knorr, R. Ng, and V. Tucakov, "Distance-based outliers: Algorithms and applications," VLDB Journal, 2000.

[6] M. M. Breunig, H.-P. Kriegel, R. T. Ng, and J. Sander, "LOF: Identifying density based local outliers," presented at ACM SIGMOD International Conference on Management of Data, 2000 
[7] S. Papadimitriou, H. Kitawaga, P. Gibbons, and C. Faloutsos, "LOCl: Fast outlier detection using the local correlation integral," presented at International Conference on Data Engineering, 2003

[8] Z. He, X. Xu, J. Huang, and S. Deng, "FP-Outlier: Frequent Pattern Based Outlier Detection", Computer Science and Information System (ComSIS'05)," 2005

[9] Shu Wu and Shengrui Wang, "Information-Theoretic Outlier Detection for Large-Scale Categorical Data, IEEE Transactions on Knowledge Engineering and Data Engineering,2011

[10] Frank, A. \& Asuncion, A. (2010). UCI Machine Learning Repository [http://archive.ics.uci.edu/ml]. Irvine, CA: University of California, School of Information and Computer Science.

[11] LakshmiSreenivasaReddy.D, .B.RaveendraBabu and A.Govardhan, "Outlier Analysis of Categorical Data using NAVF"', Informatica Economica vol 17, Cloud computing issue 1, 2013.

[12] LakshmiSreenivasaReddy.D, B.RaveendraBabu "Outlier Analysis of Categorical Data using FuzzyAVF", presented at IEEE international conference ICCPCT-2013, pp 1259-1263.

[13] LakshmiSreenivasaReddy.D, B.RaveendraBabu and A.Govardhan, "A Novel Approach to Find Outliers in Categorical Dataset" presented at Elsevier AEMDS-2013

[14] R. Agrawal and R. Srikant. "Fast Algorithms for Mining Association Rules in Large Databases." Proceedings International Conference on Very Large Data Bases, 1994, pp. 487-499.

[15] LakshmiSreenivasaReddy.D, .B.RaveendraBabu and A.Govardhan, "A model for Improving Classifier Accuracy for Categorical data using Outlier Analysis", International Journal of Computers and Technology" vol 7, 2013.

\section{THE AUTHORS}

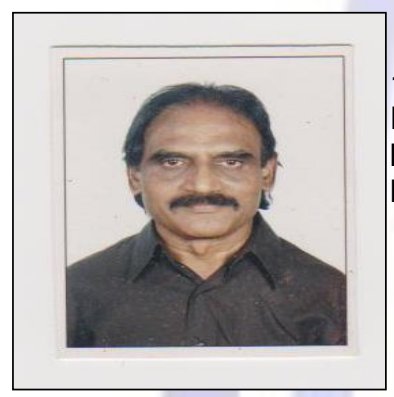

Mr. Mudimbi.Krishna Murthy did His M.C.A in first Class in 2003 from MKU Madurai. He Has 15 years of technical experience in Computer Science and Engineering at School of Information Technology (SIT), Jawaharlal Nehru Technological University Hyderabad, India. He has five research papers at international and national conferences. His area of research is Data Mining and Information Retrieval Systems.

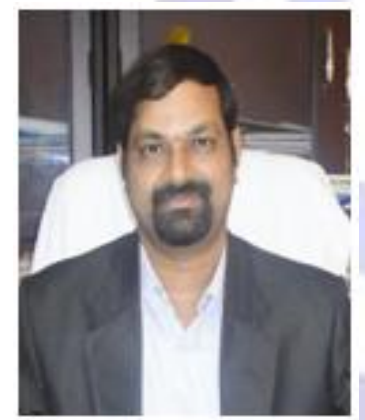

Dr.A.Govardhan is presently a Professor of Computer Science \& Engineering and Director of Evaluation, Jawaharlal Nehru Technological University Hyderabad (JNTUH), India. He did his B.E(CSE) from Osmania University College of Engineering, Hyderabad in 1992, M.Tech from Jawaharlal Nehru University(JNU), New Delhi in 1994 and Ph.D from Jawaharlal Nehru Technological University, Hyderabad in 2003. He is a recipient of several International and National Awards including A.P. State Best Teacher Award, Bharat Seva Ratna Puraskar, CSI Chapter Patron Award, Bharat Jyoti Award and Mother Teresa Award for Outstanding Services, Achievements, Contributions for Meritorious Services, Outstanding Performance and Remarkable Role in the field of Education and Service to the Nation. He is a Chairman and Member on several Boards of Studies of various Universities. He is the Chairman of CSI Hyderabad Chapter. He is a Member on the Editorial Boards for Eight International Journals. He is Member of several Advisory Boards and Committee Member for several International and National Conferences. He has guided $14 \mathrm{Ph} . \mathrm{D}$ theses and he has published 152 papers at International/National Journals/Conferences including IEEE, ACM, Springer and Elsevier. He has delivered more than 35 Keynote addresses and invited lectures. He served as Principal, Head of the Department and Students' Advisor. He is a member in several Professional and Service oriented bodies. His areas of research include Databases, Data Warehousing \& Mining and Information Retrieval Systems.

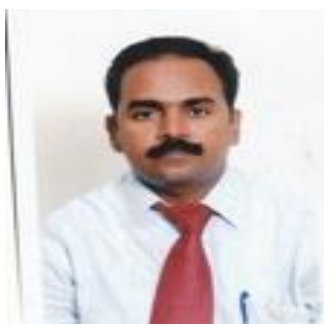

Mr.Lakshmi Sreenivasareddy.D obtained his Masters degree from Jawaharlal Nehru Technological University Hyderabad (JNTU). He is pursuing his Ph.D in Computer Science and Engineering in JNTUH, Hyderabad. He is currently heading the Department of Computer Science \& Engineering, RISE Gandhi Groups of Institutions Ongole. He has 10 years of teaching experience. His area of interest is Data Warehousing \& Mining. 